\title{
Smooth Motion Profile for Trajectory Planning of a Flexible Manipulator
}

\author{
Sameh Zribi ${ }^{\mathrm{a}}$, Marouene Mejerbi ${ }^{\mathrm{a}}$, Hatem Tlijani ${ }^{\mathrm{a}}$, Jilani Knani ${ }^{\mathrm{a}}$, Vicenc Puig ${ }^{\mathrm{b}}$ \\ ${ }^{\text {a }}$ Research Laboratory LARA in Automatic Control, National Engineering School of Tunis (ENIT), University of \\ Tunis el Manar, Tunis, Tunisia. \\ ${ }^{\mathrm{b}}$ Department of Automatic Control (ESAII), University Polytechnic of Catalonia, Barcelona, Spain.
}

\begin{abstract}
This paper deals with the problem of trajectory generation with a motion law in joint space for a flexible singlelink manipulator. To this aim, we propose a smooth motion profile based on polynomial function for a flexible manipulator. This motion law is tested with a dynamic model of the manipulator that is controlled using a model-free approach so that the robot can follow the desired trajectory.
\end{abstract}

Keywords-Trajectory generation; Flexible manipulator; Motion law; Dynamic model; Model-free control

\section{INTRODUCTION}

Trajectory generation is a very important topic of research in robotics. The planning of movements determines the robot instructions to realize the desired task. A rich scientific literature is devoted to this subject as e.g. ([1], [2]). The motion law plays a very important role either to obtain certain performances such as the time optimization, to avoid residual vibrations and to optimize the energy or to choose the actuators needed to perform the rotational and translational movements.

For trajectory generation, we need a motion profile or a motion law, so we need a mathematical function that describes the path of the robot between two positions. The final result of a motion planning problem is thus a trajectory that will then serve as an input to the real-time position/velocity controllers. This mathematical function can be a parabolic function [3], a trigonometric function, or a polynomial function [4]. Regardless of the chosen function, the selected trajectory has to satisfy some conditions that ensure the continuity of movement and that it is sufficiently configurable.

In spite of the important results obtained so far, one can distinguish two open issues: First, the case of the flexible robots and their need for the performances of the law of movement should be studied. Second, the optimization considers only one parameter that is the time, the energy or the limitation of the jerk (the derivative of the acceleration) and the snap (the derivative of jerk). The generalization of the results of motion law existing for rigid mechanical structures to flexible ones considering that the response of the system can lead to indescribable effects such as residual vibration. Thus, it is important to develop a model that takes into account the complex dynamics of the flexible systems.

The polynomial functions remain a solution which proved their effectiveness to satisfy the need to generate a soft trajectory. This type of trajectory called limited jerk trajectory is essential in order to have continuous torque. This softness of trajectory poses a problem of time optimization since there is no saturation of the actuators during the movement. Hence, there is a need to find the optimal duration while maintaining the smoothness of the trajectory. The proposed motion law is a polynomial of degree 7, but unlike classical approaches, the duration of motion is optimal by a kinematic scaling.

The main goal of this work is to determine a smooth motion profile in order to avoid the excitation of the normal modes of the mechanical structure of the robot and to avoid the phenomenon of resonance. For this purpose, this paper deals with the issue of trajectory generation with a motion law in joint space for a flexible single-link manipulator. To this aim, we propose a smooth motion profile based on polynomial function for a flexible manipulator. This motion law is tested with a dynamic model of the manipulator that is controlled using a model-free approach so that the robot can follow the desired trajectory.

The remainder of this paper is organized as follows: Section II presents a general overview about trajectory generation. Section III presents the proposed smooth motion law. Section IV is dedicated to the motion control by using a model-free controller. Simulation results are presented in Section V. Finally, concluding remarks are given in Section VI.

\section{TRAJECTORY GENERATION OVERVIEW}

The choice of the profile is strongly related to the desired performance of a robot. As the first techniques of trajectory planning, algorithms operating at minimum time were closely related to the need for increased productivity in the industrial field. This kind of motion profiles called bang-bang were formulated by Hermes using the mathematical optimal principle [3]. The transition from a beginning position to a terminal position in minimum time requires the use at any time of the maximum power leading to the saturation of the actuators. This is why for a bang-bang motion profile, the saturation of the system control should be variable, or one of its derivatives should be required, by switching several times between the maximum to the minimum allowable levels.

However, the excitation degree of vibrational modes of the considered robot was directly related to the regularity order of motion profiles. A trajectory causing high and discontinuous 
acceleration provides a high and vibrational excitation of some joints or all the structure of the robot during the motion. So, it is important to ensure a motion profile characterized by its continuous speed. As an improvement in the bang-bang profile, the trapezoidal profile allows producing a continuous motion speed which guarantees a minimum time by saturating both acceleration and speed.

In order to minimize the vibrations of the terminal elements, a constraint can be now conventionally added to the acceleration's derivative (Jerk) [4]. Another motion profile formulated by Hermes using mathematical principle is recommended to remedy the s-curve discontinuity. As described in [5], the trapezoidal motion profile is characterized by a jerk having large peaks, unlike the s-curve motion profile which exhibits a finite jerk distributed over a period of time.

Some applications need more smoothness in motion as in the case of the complex flexible arm mechanical mechanisms, for example, the motion trajectory planning may involve polynomial or trigonometric relationships. Our purpose is to achieve a motion with continuous snap (the jerk's derivative). In this case, the motion laws are presented by the sinusoidal profiles being characterized by a continuous motion during snap, jerk, and acceleration. However, it is necessary to choose the sinusoidal function and its parameters according to the joint position of departure and that of arrival. The three most common methods of polynomial profiles are linear interpolation, the third-degree polynomial interpolation, and the fifth-degree polynomial interpolation.

\section{SMOOTH Motion LAW}

Once the desired trajectory has been planned, it is frequently required to scale it in order to verify the constraints on the actuation system emerging in terms of saturations. In our work, we will consider the kinematic scaling in which the trajectory requires the satisfaction of the constraints on the maximum acceleration and velocity [6].

In order to kinematically scale the desired trajectory, it is convenient to represent this trajectory in a parametric form using a function of a suitable normalized parameter $\sigma=\sigma(t)$.

Let consider the trajectory $q(t)$ defined between two points $q_{i}$ and $q_{f}$ varying in a period of time $T=t_{f}-t_{i}$. This trajectory can be expressed in normalized form as follows:

$$
q(t)=q_{i}+h \sigma(\tau)
$$

where $h=q_{f}-q_{i}$ and the normalized time is given by $\tau=\frac{t-t_{i}}{T}$, so $0 \leq \tau \leq 1$ then $0 \leq \sigma(\tau) \leq 1$.

The maximum values of velocity, acceleration and jerk can be obtained in correspondence to the maximum values of the derivatives of functions $\sigma(t)$. By varying the period of time $T$ of the desired trajectory $q(t)$, a satisfaction of the constraints on the kinematic saturations will be possible. This trajectory is parametrized using seventh-degree polynomial function expressed as follows ([1], [7])

$$
\sigma(t)=a_{7} \tau^{7}+a_{6} \tau^{6}+a_{5} \tau^{5}+a_{4} \tau^{4}+a_{3} \tau^{3}+a_{2} \tau^{2}+a_{1} \tau+a_{0}
$$

The constraints allowing the satisfaction of the required angles with a smooth motion can be described by the boundary conditions as follow

$$
\begin{aligned}
& q(0)=q_{i} ; \quad \dot{q}(0)=0 ; \quad \ddot{q}(0)=0 ; \quad q\left(\frac{\left(t_{f}\right)}{2}\right)=\frac{q\left(t_{f}\right)}{2} \\
& q\left(t_{f}\right)=q_{f} ; \quad \dot{q}\left(t_{f}\right)=0 ; \quad \ddot{q}\left(t_{f}\right)=0
\end{aligned}
$$

Let define respectively with $v_{0}, v_{1}, j_{0}$ and $j_{1}$ the velocities and jerks in the initial and final positions.

After applying these conditions, the coefficients of the seventh-degree polynomial function are deduced as follows

$$
\begin{gathered}
a_{0}=q_{i} ; a_{1}=v_{0} ; a_{2}=\frac{a_{0}}{2} ; a_{3}=\frac{j_{0}}{6} \\
a_{4}=\frac{\left[210 h-T\left(\left(4 j_{0}+j_{1}\right) T^{2}+\left(30 q_{i}-15 v_{0}\right) T+120 v_{0}+90 v_{l}\right)\right]}{\left(6 T^{4}\right)} \\
a_{5}=\frac{-\left[168 h-T\left(\left(2 j_{0}+j_{1}\right) T^{2}+\left(20 q_{i}-14 v_{0}\right) T+90 v_{0}+78 v_{1}\right)\right]}{\left(2 T^{5}\right)} \\
a_{6}=\frac{\left[420 h-T\left(\left(4 j_{0}+3 j_{1}\right) T^{2}+\left(45 q_{i}-39 v_{0}\right) T+216 v_{0}+204 v_{1}\right)\right]}{\left(6 T^{6}\right)} \\
a_{7}=\frac{-\left[120 h-T\left(\left(j_{0}+j_{1}\right) T^{2}+\left(12 q_{i}-12 v_{0}\right) T+60 v_{0}+60 v_{l}\right)\right]}{\left(6 T^{7}\right)}
\end{gathered}
$$

\section{Model-Free Control of FleXible Manipulator Robot}

\section{A. Description and Dynamic Modeling of Flexible Single- Link Manipulator}

Before presenting the modeling steps of flexible single-link manipulator robot, let begin with its description. It is composed of a flexible single-link arm, pivotally related to one extremity with a base via a rotary motion $\tau(t)$ which is achieved by a DC motor and fixed on its second one a mass load ( [8], [9]).

Based on a simplified representation of the flexible singlelink mechanism described in Figure 1, where

- $I$ is the area moment,

- $E$ is the modulus of Young,

- $I_{m}$ is the inertia moment of the base,

- $A$ is the section of the single-link arm, 
- $\quad \rho$ is the mass density per unit volume,

- $\alpha$ is the revolution angle of the manipulator relative to the origin,

- $\theta(t)$ is the angular translation of the link in the $x_{0} o y_{0}$ coordinates,

- $g$ is the centre of gravity,

- $\quad x o y$ and $x_{0} o y_{0}$ are respectively the moving frame and the stationary one,

- $L$ is the length of the single-link arm where the elementary lengths $L_{1}$ and $L_{2}$ verify $L_{2}=2 L_{1}$,

- $W(x, t)$ is the elastic deflection of the flexible manipulator at a distance $x$ from the base which is measured along the $o x_{1}$ axis.

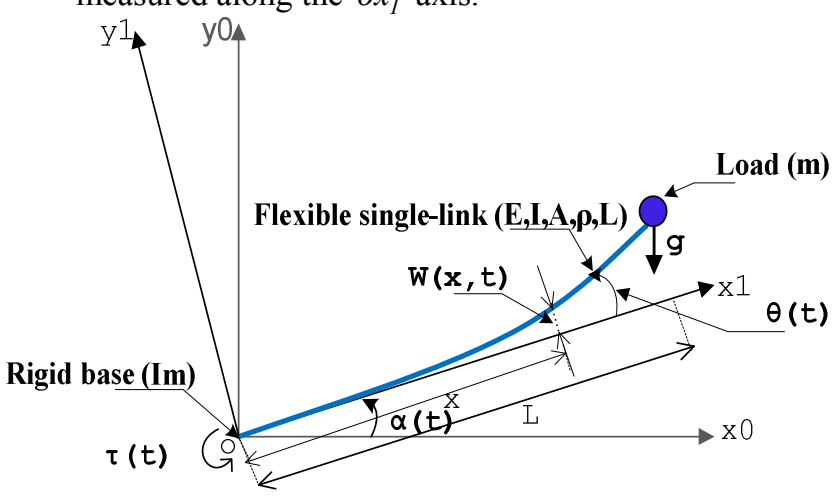

Fig. 1. Flexible manipulator robot with load scheme.

From [10], the following modelling assumptions are considered:

- The shear strain, the rotational inertia and the effect of the axial force are negligible,

- The length of this single-link manipulator is supposed to be constant to avoid the problems appeared when this length is variable,

- Its depth is supposed to be smaller than its length allowing that the arm can vibrate in the horizontal direction.

The dynamic modelling of the considered mechanism presented by the Bernoulli-Euler beam is performed using the Euler-Lagrange formulation and the finite elements approach ([11-16]). Remember that in all expressions, a number between parentheses in the power of such a function means a time derivative.

The dynamic equation described the dynamic of the considered mechanism is given by

$$
M q^{(2)}{ }_{1}+K q_{1}=\tau
$$

where $M, K, \tau$ and $q_{1}$ represent respectively the system mass matrix, the system stiffness matrix, $\tau$ the torque applied to the manipulator, and the vector of generalized coordinates ([13], [17]).

\section{B. Model-Free Control}

The principle of the model-free control also called ultralocal model control consists in a local modeling, instantly updated using only the information received from the inputoutput behavior. In the first step, let begin with the representation of a linear or nonlinear system by an unknown differential equation as follows

$$
H\left[y(t), y^{(1)}(t), \ldots, y^{(a)}(t), u(t), u^{(1)}(t), \ldots, u^{(b)}(t)\right]=0
$$

where $H$ presents appropriately a smooth function of its arguments, $u$ and $y$ represent respectively the input and the output of the system. We suppose that, for an integer, $0<v<a, \frac{\partial H}{\partial y^{(v)}} \neq 0$.

In the second step, using the implicit function theorem we obtain an equation which approximately details the inputoutput behaviour as follows

$$
y^{(v)}=g\left[t, y, y^{(1)}, \ldots, y^{(v-1)}, y^{(v+1)}, \ldots ., y^{(a)}, u, u^{(1)}, \ldots . ., u^{(b)}\right]
$$

Based on the input and the output measurements, the considered control try to estimate the parameters to obtain a good tracking of desired trajectory. Then, a numerical model of the system can be constructed as follows

$$
y^{(v)}=F+\alpha u
$$

where $v$ presents the order of derivative that should be equal to 1 or $2, \alpha$ are a non-physical constant and undefined previously parameter and $F$ present the model-free model parameters containing the structural information, including disturbances and their derivatives.

In the third step, the estimation of $F$ is performed using an on-line estimation approach called an algebraic derivation method ([18], [19]) which is given by

$$
\hat{F}_{k}=\hat{y}_{k}{ }^{(1)}-\alpha u_{k-1}
$$

where $u_{k-1}$ is the control input, $\hat{y}_{k}^{(1)}$ is the estimate of first derivative of the output of the considered system.

Finally, for $v=1$, the desired closed-loop behavior can be deduced thanks to the structure of PID controller suggested in [18]. So, the control signal can be rewritten by

$$
u(t)=\frac{-\hat{F}(t)+y^{d^{(l)}}(t)+K_{P} e(t)+K_{I} \int e(t)+K_{D} e^{(l)}(t)}{\alpha}
$$

where $K_{P}, K_{I}$ and $K_{D}$ are the tuned gains of the PID controller that are manually adjusted, $y^{d}(t)$ is the output of reference trajectory, $y^{d^{(1)}}(t)$ is the derivative of $y^{d}(t), e(t)$ 
is the tracking error that is evaluated asthe difference between $y^{d}(t)$ and $y(t)$.

In closed-loop and without taken into account noise and disturbances, the block diagram of the flexible single-link manipulator controlled by ultra-local model control is presented in Figure 2.

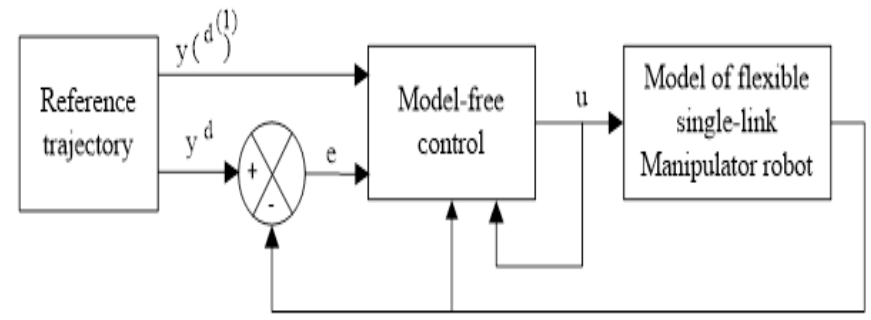

Fig. 2. Representation of model-free control applied to flexible single-link robot

\section{Simulations Results AND Discussion}

After obtaining the dynamic model of flexible single-link manipulator robot described by (9) and the model-free control described by (14), the torque will be applied to each joint for a seventh-degree polynomial motion profile. The numerical

Table 1. Dynamic parameters of flexible manipulator.

\begin{tabular}{|c|c|c|}
\hline Parameter & Value & Unit \\
\hline$E$ & 196 & $\mathrm{GPa}$ \\
\hline$I$ & $1.67 \times 10^{-12}$ & $\mathrm{~m}^{4}$ \\
\hline$A$ & 0.02 & $\mathrm{~m}^{2}$ \\
\hline$\rho$ & 3.25 & $\mathrm{Kg} / \mathrm{m}^{3}$ \\
\hline$I_{m}$ & 0.04 & $\mathrm{Kg} \cdot \mathrm{m}^{2}$ \\
\hline$L_{1}$ & 0.15 & $\mathrm{~m}$ \\
\hline$L_{2}$ & 0.30 & $\mathrm{~m}$ \\
\hline$m$ & $5 \times 10^{-3}$ & $\mathrm{Kg}$ \\
\hline
\end{tabular}

Table 2. Model-free controller parameters.

\begin{tabular}{|c|c|c|}
\hline Parameter & Value & Unit \\
\hline$K_{P}$ & 3 & - \\
\hline$K_{I}$ & $15 \times 10^{-2}$ & - \\
\hline$K_{D}$ & $10^{-3}$ & - \\
\hline$\alpha$ & $5 \times 10^{-1}$ & - \\
\hline
\end{tabular}

values of parameters of the considered mechanism and that of the considered control are respectively detailed in Table 1 and in Table 2.

The boundary conditions related to the seventh-degree polynomial function is described as follows

$$
\begin{aligned}
& q(0)=10 ; \quad \dot{q}(0)=0 ; \quad \ddot{q}(0)=0 ; \quad q\left(\frac{\left(t_{f}\right)}{2}\right)=\frac{q\left(t_{f}\right)}{2} \\
& q\left(t_{f}\right)=50 ; \quad \dot{q}\left(t_{f}\right)=0 ; \quad \ddot{q}\left(t_{f}\right)=0
\end{aligned}
$$

where $t_{f}=2.455$. After ignoring the jerks and velocities in initial and final positions, the coefficient of this motion profile are given by

$$
\begin{aligned}
& a_{0}=10 ; \quad a_{1}=0 ; \quad a_{2}=5 ; \quad a_{3}=0 \\
& a_{4}=30.4362 ; \quad a_{5}=-31.1529 \\
& a_{6}=10.8200 ; \quad a_{7}=-1.2771
\end{aligned}
$$

After applying the torque to second joint considering a seventh-degree polynomial profile, the response of flexible manipulator mechanism is presented by the variation of joint acceleration with respect to the time as described by Figure 3 .

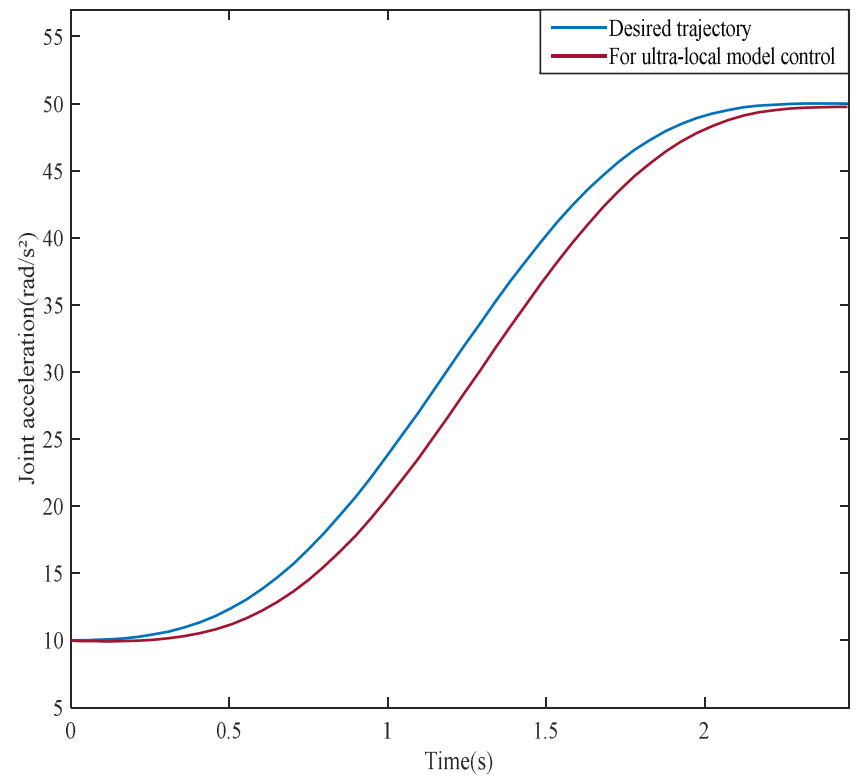

Fig. 3. System response for torque applied to joint for seventh-degree polynomial profile

We note that the acceleration is chosen as a proof of smoothness of motion profile because as presented in (9), the variation of the torque $(\tau)$ is principally proportional to the variation of the term $\left(M q^{(2)_{1}}\right)$ which contains the acceleration.

It is clear that after a small time difference, the system response follows a smooth trajectory described by the seventhdegree profile considering the flexibility of the structure. Thus, the goal of this work has been achieved by improving the smoothness of the desired trajectory as an input signal and by ensuring optimality in terms of time.

Take for example the case of the fifth-polynomial motion profile detailed in [19], by comparing this motion profile with that of our case (seventh-polynomial motion profile), we note that the first profile whose acceleration curve contains fluctuations requires more time to reach the desired endpoint while the second profile whose acceleration curve does not contain any fluctuation allows reaching the desired endpoint in a minimum time. Hence, it can be said that the seventhpolynomial motion profile is optimal in time with respect to the fifth-polynomial motion profile for example. 


\section{CONCLUSION}

This paper has considered problem of obtaining a smooth motion profile for trajectory planning of a flexible manipulator robot. For this purpose, this flexible mechanism is dynamically modelled considering several motions profiles.

The main contribution of this paper is to describe a new joint space trajectory generation method using a single polynomial function. The single link flexible robot requires a smooth and continuous motion as input to improve the performance of the flexibility structure. To this aim, a seventhpolynomial was developed by imposing seven boundaries conditions. With this new approach of trajectory generation, the associate jerk function will have a finite spike along the period time. Consequently, the vibration phenomena are reduced and the considered robot ensuring the following trajectory.

In this work, we are interested in studying the system behavior for a smooth and time optimal input profile presented by seventh-polynomial motion profile. In the future work, we will focus on improving the system's response in terms of saving energy by optimizing the parameters of this polynomial motion profile.

\section{REFERENCES}

[1] L Biagiotti and C Melchiorri, "Trajectory Planning for Automatic Machines and Robots," Springer- Verlag-Berlin- heidelburg- 2009.

[2] W. Khalil and E. Dombre, "Modelisation, identification and control of robots," Hermes Penton Science, London, p. 480, 2002.

[3] H Hermes and J. P. Lasalle, "Functional analysis and time optimal control," New York, Academic Press, 1969.

[4] K. J. Kyriakopoulos and G. N. Saridis, "Minimum jerk path generation," IEEE International Conference on Robotics and Automation, 1988, 1988.

[5] P. H. Meckl, P. B. Arestides, and M. C. Woods, "Optimized S-Curve Motion Profiles for Minimum Residual Vibration," Proceeding of the American Control Conference, Pennsyl-vania, USA, pp. 26272631,1998 .

[6] L. Biagiotti and C. Melchiorri, "Trajectory Planning for Automatic Machines and Robots," Springer-Verlag Berlin Heidelberg, pp. 15-47, 2008.
[7] R. L. Williams, "Improved robotics joint-space trjectory generation with via point," International Design Engineering Technical Conferences \& Computers and Information in Engineering Conference, Washington, USA,28-31 August, 2011.

[8] W. Yang Liu and Y. Jia, "Adaptive Consensus Control for Multiple Euler-Lagrange Systems with External Disturbance", International Journal of Control, Automation, and Systems, Vol. 15, No. 1, pp.205211, 2017.

[9] L. Meirovitch, "Elements of Vibration Analysis", library of congress cataloging-in-publication data, 1982.

[10] S. Zribi, H. Tlijani, J. Knani and V. Puig, "On the dynamic modeling and simulation of rigid-flexible manipulator robot using several inputs," in International Conference on Control, Decision and Information Technologies, Barcelona, Spain, pp. 579-584, 2017.

[11] S. Zribi, H. Tlijani, J. Knani and V. Puig, "Impact of external disturbance and discontinuous input on the redundant manipulator robot behaviour using the linear parameter varying modelling approach," International Journal of Advanced Computer Science and Applications, Vol. 8, No. 10, 2017.

[12] H. Roy Pota, "Finite-element/Lagrange Modeling and Control of a Flexible Robot Arm," IFAC World Congress, Vol. 9, 1990.

[13] M. Mejerbi, S. Zribi and J. Knani, "Dynamic Modeling of Flexible Manipulator Based on a Large Number of Finite Elements," International Journal of Advanced Computer Science and Applications, Vol. 8, No. 12, 2017.

[14] W. Khalil and E. Dombre, "Dynamics of robots with complex structure", in Modeling, Identification and Control of Robots, 2002.

[15] J. Knani, "Dynamic modelling of flexible robotic mechanisms and adaptative robust control of trajectory computer simulation-Part I," Applied Mathematical Modeling, Vol. 26, No. 12, p. 12, 2002.

[16] E. Bayo, "A Finite-Element Approach to Control the End-Point Motion of a Signle-Link Flexible Robot," journal of robotic system, Vol. 4, pp. 63-75, 1986.

[17] S. Zribi, M. Mejerbi, H. Tlijani and Jilani Knani, " Comparison between motions profiles applied to flexible manipulator arm," 3rd International Conference on Automation, Control Engeneering and Computer Science, pp. 565-571, 2016.

[18] M. Fliess and C. Join, "Model-free control," International Journal of Control, Vol. 68, No. 12, 2013.

[19] S. Zribi, W. Boucetta, H. Tlijani, J. Knani and V. Puig, "Comparison between ultra-local model and Auto-tuning PID controls strategies applied to the redundant manipulator robot for several inputs," International Conference on Sciences and Techniques of Automatic control and computer engeneering STA, Vol. 11, No. 2, 2017. 